\title{
La tarea (cada vez más impropia) del traductor. Un breve panorama del ejercicio de la traducción en Canadá hoy
}

\author{
Claudia LUCOTTI \\ Universidad Nacional Autónoma de México
}

\begin{abstract}
Este texto ofrece un breve panorama de las discusiones y los cambios más importantes que se han dado a partir de los setentas en Canadá en el mundo de la traducción literaria, sobre todo del francés al inglés. Comienzo con el papel destacado que tuvieron las traductoras feministas en Quebec, continúo con el caso de las traducciones del francés de la clase trabajadora (joual) y concluyo con un estudio sobre las aportaciones más recientes que ha realizado Sherry Simon de lo que acontece en este universo, así como sobre su estiramiento de los límites tradicionalmente aceptados para esta actividad.

PALABRAS ClAVE: traducción, literatura, Canadá, inglés, francés.

This text offers a brief survey of the main discussions and changes that have taken place, since the seventies, in Canada in the world of literary translation, particularly of French literature into English. I begin with the important role feminist translators played in Quebec, then I discuss the translation of working class joual, and I end up working on Sherry Simon's most recent contributions to the study of what is happening today in this field in Canada and on her stretching of the traditionally accepted limits of this activity.
\end{abstract}

KEY WORDS: translation, literature, Canada, English, French.

Para Sherry Simon, con afecto y gratitud

Es indiscutible el hecho de que en años recientes, debido a diversas y complejas razones, han comenzado a soplar vientos nuevos en numerosas áreas de estudio dentro de las humanidades, lo cual ha resultado sumamente renovador y estimulante para muchos académicos y académicas. Estas nuevas tendencias buscan cuestionar las fronteras, hasta hace poco consideradas infranqueables, que existen entre las distintas áreas del conocimiento. Asimismo, buscan tender puentes novedosos y cada vez más necesarios, entre nuestras tareas universitarias y nuestras vidas personales y políticas, dando pie a nuevos proyectos que obligan a reformular prácticas tradicionales. Fueron quizá los estudios de la mujer - luego de género - seguidos por los poscoloniales donde se vivieron estos cambios con mayor intensidad, ya que estas nuevas reflexiones teóricas 
implicaban tomar en cuenta los posicionamientos y las experiencias concretas tanto de los autores como de los lectores, así como replantearse antiguas formas de trabajo. A su vez, esto contribuyó a entender con creciente claridad que todo texto es un constructo que responde a coyunturas y juegos de poder particulares, y que incluso son estas mismas coyunturas y juegos de poder los que definen las reglas que gobiernan el modo de proceder dentro de las áreas de estudio particulares y, también, imponen límites entre ellas. Unos años después, los estudios sobre la traducción así como su práctica misma fueron igualmente sujetos a revisiones interesantes en las que las fronteras, tan claramente establecidas de forma previa, comenzaron a ser cuestionadas y a flexibilizarse.

Considero, pues, que en una publicación destinada a reflexionar sobre la noción de frontera como demarcación entre culturas y saberes, y sobre las apuestas políticas derivadas de horizontes interpretativos que surgen a partir de conceptualizar la frontera como espacio permeable y poroso, no sale sobrando un texto que ofrezca un breve panorama de las discusiones y los cambios tan sugerentes que se están dando dentro del universo de la traducción. En este caso, me voy a concentrar en lo que sucede actualmente en Canadá pues se trata de un país en donde la traducción ha sido siempre una actividad central debido, sin duda alguna, a su historia tan particular.

En el apretado panorama que ofrezco a continuación, quiero empezar por mencionar el papel tan destacado que tuvieron a partir de los setentas - y siguen teniendo - las feministas, sobre todo las más relacionadas con los movimientos literarios y culturales de Quebec. En décadas recientes, algunas autoras en lengua francesa de esta región comenzaron a producir nuevas formas de escritura que se inscriben dentro de lo que se ha llamado écriture féminine; ${ }^{1}$ paralelamente, dichas autoras fueron estableciendo vínculos con escritoras de habla inglesa, lo cual resultó en varios proyectos que, aunque eran de diversa índole, estaban ligados de algún modo u otro a la traducción y buscaban trasladar del francés al inglés los aspectos de mayor interés y relevancia de estos escritos novedosos. Dentro de este campo destacan los nombres de Barbara Godard y Susanne de Lotbinière-Hardwood. Y si bien es cierto que en cuestiones teóricas relativas a la traducción ya había aportaciones interesantes como las de la inglesa Susan Bassnett, en cuestiones prácticas estas canadienses realizaron experimentos innovadores que abrieron brecha. Ante todo, se replantearon las rígidas convenciones que supuestamente debían acatarse como traductores ideales invisibles en las relaciones que establecían con lo que traducían. Tanto Godard como De Lotbinière-Hardwood optaron por prestar cada vez más atención a cómo sus posicionamientos específicos como

\footnotetext{
${ }^{1}$ Término primeramente propuesto y empleado por la crítica feminista francesa de los años setentas, particularmente Hélène Cixous, Luce Irigaray y Julia Kristeva, y que supone la existencia de una escritura que puede calificarse como "femenina" aunque no es producida únicamente por mujeres. Dicha forma de escritura se opone a y subvierte la lógica "masculina" o patriarcal que rige el lenguaje y las estructuras simbólicas de poder, dentro de los cuales predomina una visión jerarquizada y esencialista de las diferencias sexuales.
} 
mujeres canadienses de extracciones diversas en cuanto a raza, religión y preferencia sexual influían de manera directa en sus procesos y productos de traducción, lo cual puso nuevamente en el centro de la discusión tanto la viabilidad de una única traducción ideal así como la neutralidad, invisibilidad y pasividad del traductor.

Barbara Godard ${ }^{2}$ ha destacado no sólo debido a su interés y entrega por traducir y difundir las obras de escritoras originarias de Quebec, sino porque a través de numerosos escritos ha reflexionado, de modo crítico, personal y comprometido, en torno a su labor como traductora, lo cual significa también considerar que el/la traductor/a merece un espacio reconocido. Para ella, la traducción, lejos de reducirse a una búsqueda casi automática de correspondencias entre palabras de dos distintas lenguas, se define como un ejercicio que procura reconstituir, de manera activa e involucrada, un amplio universo textual, vivo y marcado por un sistema cultural determinado, en otro universo textual, marcado éste por un sistema cultural diferente. Asimismo, Godard plantea que la experiencia y el posicionamiento específico de cada autor/a y traductor/a también juegan un papel fundamental en todo este proceso. Para ella, uno de los ejemplos más claros de lo anterior es la traducción de textos que pertenecen a la écriture féminine, es decir, de textos que invitan a numerosas interpretaciones y cuya producción de significados se da a través de mecanismos múltiples, y en donde, por lo tanto, no es siquiera posible hablar de una correspondencia fiel entre palabras (Simon, 1996: 12 13). Esto la lleva a revisar las características tradicionales tanto del proceso de traducir como de los productos resultantes. Así pues, para ella, autor/a y traductor/a se relacionan de manera recíproca y visible en un proceso de determinación de significados, tal como el texto original y la traducción se relacionan en un ir y venir que da por resultado una iluminación y un enriquecimiento mutuos.

Su traducción de la novela de Nicole Brossard Picture Theory ${ }^{3}$ - texto que se caracteriza de modo notable por su carácter interdiscursivo, ${ }^{4}$ por estar anclado dentro de un contexto cultural y social específico, y por invitar al lector a contribuir en la generación de significados - se convirtió en un caso muy representativo de lo anterior. En su libro Gender in Translation (1996), Sherry Simon le dedica un apartado a la labor de Godard como traductora. De manera muy atinada y precisa, Simon destaca la contribución de Godard a la traducción feminista por explorar estrategias que ponen de

\footnotetext{
${ }^{2}$ Además de desempeñarse como académica, teórica y editora, se ha ocupado de traducir al inglés a numerosas autoras canadienses francófonas como Louky Bersianik, Yolande Villemaire, Antonine Maillet, France Théoret y la célebre Nicole Brossard, de quien se hablará más adelante. Como teórica ha publicado: "Une littérature en devenir: la réécriture textuelle et le dynamisme du champ littéraire. Les écrivaines québécoises au Canada anglais", en Voix et images, XXIV.3 (72), 1999, pp. 495-527; "Deleuze and Translation", en Parallax, 6.1, 2000, pp. 56-81, y "French-Canadian Writers in English Translation", en Encyclopedia of Literay Translation into English. Ed. de Olive Classe. Londres, Fitzroy Dearborn Publishers, 2000, pp. 477-82.

${ }^{3}$ Publicada con ese mismo título en francés: Picture Theory. Montreal, Éditions Nouvelle-Optique, 1982; la traducción de Godard es del año 1991: Picture Theory (Montreal, Guernica).

${ }^{4} \mathrm{La}$ interdiscursividad en el texto de Brossard consiste en la interacción de discursos sociales y culturales específicos con distintas fuentes literarias o filosóficas, como escritos de Wittgenstein, Gertrude Stein, Joyce y Djuna Barnes, entre otros (Simon, 1996: 23).
} 
manifiesto la posición discursiva de quien traduce. Comenta, además, sobre la decisión de Godard de llevar un diario durante la traducción de Picture Theory, en el cual intenta reconstruir el proceso creativo de la escritura de Brossard, pero también dar cuenta de sus propias operaciones mentales, revelando así el carácter asociativo de la labor de traducir. Para Godard, la traducción consiste en darle continuidad a los mecanismos involucrados en la producción de sentidos del texto que se está traduciendo, sin pasar por alto cómo intervienen también otros textos y prácticas discursivas culturalmente específicas y sin omitir, por último, la plasticidad misma del lenguaje, tal como se manifiesta en el ritmo o los juegos de palabras (22-24). En palabras de Simon:

The challenge of translating Picture Theory is to orchestrate the repeated themes and developments in the same way as is done in the original, reactivating fragments and themes. Godard emphasizes the "transferential process" of translation, the reading subject becoming the writing subject. Like the author, the translator uses disjunctive strategies, breaking with a unified language. For example, Brossard uses English words in her French text in order to disrupt the code and to enhance the power of certain terms $(25){ }^{5}$

Simon señala que este acercamiento al texto por parte de Godard tiene como resultado que la subjetividad de la traductora se manifieste en el texto traducido, de manera que el lector o la lectora leen a "Nicole Brossard y Barbara Godard juntas" (27). Por otra parte, queda claro que si bien Brossard cuestiona en su escritura la correspondencia lineal entre objeto y palabra, Godard, por su parte, cuestiona la correspondencia lineal entre palabra (en francés) y palabra (en inglés), de modo que, en efecto, la traducción le da continuidad a las búsquedas de la escritura de Brossard, y tanto la autora como la traductora ejercen una crítica hacia el lenguaje y la traducción vistos como prácticas estables y unidireccionales. Finalmente, la misma Godard, en su diario, nos ofrece la siguiente reflexión, que bien puede servir como conclusión a esta breve sección acerca de las controversiales aportaciones prácticas que realizó a la traducción literaria en su país:

No final version of the text is ever realizable. There are only approximations to be actualized within the conditions of different enunciative exchanges. As such, translation is concerned not with "target language" and the conditions of "arrival" but with the ways of ordering relations between languages and cultures. Translation is an art of approach (apud Simon, 1996: 24). ${ }^{6}$

\footnotetext{
5 "El desafío de traducir Picture Theory consiste en orquestar los temas recurrentes y sus desarrollos de la misma manera como sucede en el original, reactivando fragmentos y temas. Godard enfatiza el 'proceso transferencial' del acto de traducir, en el cual el sujeto que lee se convierte en el sujeto que escribe. Como la autora, la traductora emplea estrategias disyuntivas que rompen con un lenguaje unificado. Por ejemplo, Brossard usa palabras en inglés en su texto, escrito predominantemente en francés, con el propósito de desestabilizar el código e intensificar el poder de algunos términos". Todas las traducciones son mías.

6 "La versión final del texto nunca será alcanzable. Sólo podemos hablar de aproximaciones realizables dentro de las condiciones de diferentes intercambios enunciativos. Como tal, la traducción no tiene como
} 
Otra traductora destacada es Susanne de Lotbinière-Hardwood, ${ }^{7}$ quien también se ocupa de cuestiones de género, textualidad e identidad, y ha realizado traducciones muy interesantes y con implicaciones de índole política en su sentido más amplio. Si bien su lengua materna es el francés, traduce literatura quebequense al inglés por considerar que debe contribuir a su difusión, sobre todo si se toma en cuenta que, en Canadá, la traducción de literatura francófona a lengua inglesa es poco equitativa con respecto a las traducciones que se llevan a cabo del inglés al francés. De Lotbinière-Hardwood piensa y justifica largamente su elección de obras a trabajar. Después de traducir textos ligados a la famosa Revolución Tranquila de los sesentas, ${ }^{8}$ comenzó a interesarse por traducir textos escritos por mujeres y, en este sentido, su labor más connotada ha sido su traducción de otro libro de Nicole Brossard, Le Désert mauve. ${ }^{9}$ Ésta es una extraordinaria obra sobre traducción en la que, como parte de la trama, la "novela" inicial que se presenta al principio del libro, aunque escrita en francés, se "traduce" al francés - lo cual también se incluye en el libro - subrayando una y otra vez el hecho de que la traducción es un acto creativo y nunca mera reproducción. Al respecto, en su artículo "Geo-graphies of Why", la traductora dice: "Imagine the challenge of translating interlingually, from French into English, a novel already translated, or re-written intralingually, from French into French, by its author Nicole Brossard" (Simon, ed. 1995: 59). ${ }^{10}$ Este hecho permitió a De Lotbinière-Hardwood realizar también una traducción muy experimental, en la que manipuló el inglés para introducir, por ejemplo, marcas de género gramatical, ausentes en dicha lengua pero centrales para el francés al igual que para el español, conservando la presencia de una serie de elementos lésbicos que hubieran quedado cancelados en una traducción más convencional.

Más adelante, de Lotbinière se pregunta si no se ha excedido en la ruptura de límites, produciendo una traducción que rebasa todas las fronteras previstas. Sin embargo, concluye su artículo diciendo: "but translating in the feminine is a political activity aimed at making the feminine, i. e. women, visible in language and in reality. Solidarity with the auther (sic) encourages me to take the same risks she did. This implies aesthetic and ethical choices" (idem.). ${ }^{11}$ De hecho, según Sherry Simon (1996: 7-8)

principal preocupación el 'lenguaje de llegada' y las condiciones del 'arribo', sino los modos de organizar relaciones entre distintas lenguas y culturas. La traducción es un arte de aproximación".

${ }^{7}$ Además de traductora, es autora del libro Re-belle et infidèle: la traduction comme pratique de réécriture au féminin / The Body Bilingual: Translation as a Rewriting in the Feminine. Montreal / Toronto, Les Éditions du Remue-ménage / Women’s Press, 1991.

${ }^{8}$ Llamada Révolution tranquille en francés, este proceso social y político tuvo como objetivo modernizar a la provincia de Quebec a través de la creación de instituciones gubernamentales para la prestación de servicios (previamente en manos de la Iglesia católica) y la implantación de reformas económicas que le dieran mayor independencia a la provincia.

${ }^{9}$ Nicole Brossard, Le Désert mauve. Montreal, L'Hexagone, 1987. Traducido como Mauve Desert (Toronto, Coach House, 1990).

10 "Imaginen el desafío de traducir interlingüemente, del francés al inglés, una novela ya traducida, o rescrita intralingüemente, del francés al francés por su autora Nicole Brossard".

11 " [...] pero traducir en femenino es una actividad política dirigida a que lo femenino (es decir, las mujeres) se vuelva visible en el lenguaje y en la realidad. Por solidaridad con la autora me atrevo a tomar 
estas discusiones y revaloraciones por parte de traductoras como Godard y De Lotbinière-Hardwood fueron de tal magnitud que tuvieron como resultado el que conceptos y ejercicios tradicionales ligados a la traducción - largamente entendidos y trabajados como relacionados con las categorías de, por ejemplo, corrección, fidelidad y autoridad, y pertenecientes a áreas de estudio claramente separadas y definidas - hayan comenzado a fundirse en lo que ella llama "una hibridación disciplinaria" emparentada ya con los estudios culturales. Así pues, las preguntas tradicionales sobre qué constituye una traducción correcta, las cuales estaban básicamente enfocadas a cuestiones de gramática y semántica, han dado pie a otras inquietudes que giran en torno a qué efectos o respuestas logra el poner en circulación una traducción en un nuevo contexto.

Otro aspecto de gran interés, aunque no siempre entendible desde México, de la traducción en Canadá es el que se liga con el joual, es decir, el francés de la clase trabajadora de Quebec, que en los años sesentas se volvió un instrumento de la población quebequense para reivindicar una muy compleja identidad propia, tanto a nivel político como cultural. El que numerosos escritores y dramaturgos incorporaran el joual a sus obras le otorgó a éste una mayor visibilidad no sólo en Quebec, sino en el Canadá de habla inglesa y en el extranjero, y es aquí donde la traducción —obviamente - resultó clave. Sin embargo, el tema es más complejo de lo que se ve a simple vista, debido a que la cuestión consta de varios niveles prácticos y éticos, y cada uno impacta en la traducción de manera distinta.

En primer lugar, tenemos la presencia de un francés que se rebelaba contra todas las reglas que normaban el francés estándar, tanto en cuestiones gramaticales como de pronunciación. Lo anterior representaba ya un gran desafío a la hora de buscar su traducción al inglés, pues había que sopesar con cuidado qué tipo de inglés permitiría reproducir el propósito deliberado de apartarse de una variante lingüística largamente aceptada como la deseable. ${ }^{12}$ Pero, en segundo lugar, tenemos el problema adicional de que el joual hacía usos frecuentes, pero muy variados, de palabras inglesas de las que se había apropiado debido a razones diversas y complejas. Dichos anglicismos siempre presentan problemas espinosos a la hora de la traducción, ya que justamente la hibridez del joual nace de las profundas tensiones que existen entre estas dos comunidades: la anglófona y la francófona. Como ejemplo de lo anterior podemos mencionar a dos escritores sobre los cuales Betty Bednarski, una reconocida traductora de Quebec, ha reflexionado en detalle. Uno es Jacques Renaud, quien incorpora expresiones como gouffebâles y mâchemallo pero siempre dentro de contextos lingüísticos y narrativos en donde se percibe un creciente desgaste, incluso derrota, del francés ante

los mismos riesgos que ella [Brossard] tomó, lo cual involucra decisiones estéticas y éticas". De Lotbinière emplea en inglés la palabra auther para preservar el término en francés auteure propuesto por las feministas en Quebec.

${ }^{12}$ Ver el capítulo "Translating Tremblay into Scots" de William Findlay, en Culture in Transit (Simon, ed., 1995, pp. 149-162), en donde Findlay describe y comenta la traducción que realizó de la obra de teatro Les belles-soeurs de Michel Tremblay. 
el avance inexorable del inglés. El otro es Jacques Ferron, un autor importante en la escena canadiense, quien se apropia del inglés pero de modo muy distinto a Renaud, ya que en su caso el francés siempre resulta poderoso y en total control, y es el inglés el que sufre el desgaste de la asimilación. Con respecto a Ferron, Bednarski llega incluso a hablar de un proceso de enquebequesimiento del inglés en donde:

\begin{abstract}
All authority was with the French text. English words, momentarily subjected to the conventions of French, were being mischievously assimilated. Ferron would have said enquébecquoisés... It was as though he was exacting a kind of playful revenge, getting his own back on a language which, in a lighthearted and fanciful text on anglicisms, he had once commented was "en train de faire une job au français" (Simon, ed. 1995: $113-14) .^{13}$
\end{abstract}

Todo lo anterior crea, como ya dijimos, numerosos problemas a la hora de traducir, sobre todo, y por razones obvias, al inglés, lo cual ha resultado en una serie de planteamientos al respecto por parte de traductores comprometidos, en donde las cuestiones ligadas a la práctica concreta de la traducción se entremezclan con reflexiones que se inscriben ya dentro de los estudios culturales, las políticas de la traducción y la política misma. Para Sherry Simon, la especificidad del lenguaje empleado en la literatura quebequense de los sesentas y los setentas busca expresar cierta especificidad cultural de la provincia (con respecto, principalmente, al resto de la población nacional), y parte del carácter transgresor de dicho lenguaje proviene precisamente del uso de palabras en inglés. Así pues, la autora se pregunta: "[w]hat would be the ethics of rerouting those English words back to a totally English context?" (1992: 171). ${ }^{14}$

En relación con esta última interrogante, ha habido, por parte de los traductores, modos de proceder diversos. Algunos de ellos, situados un poco más atrás en el tiempo, y quizá como resultado de la presión ejercida por las casas editoriales, se preocuparon especialmente por hacer que la traducción al inglés fuera accesible a sus lectores. Por tal motivo, al enfrentarse a expresiones consideradas "imposibles de traducir" privilegiaban en ocasiones el sistema de referencias y los mecanismos para la producción de significados de la lengua de llegada. Desafortunadamente, como señala Kathy Mezei, el texto originario de Quebec queda asimilado así a la literatura anglocanadiense (Simon, ed. 1995: 144-45). Es importante recalcar la gravedad de una situación como ésta, ya que justamente al traducir de forma tergiversada o incluso eliminar elementos del texto original (mis- o non-translation) que resultan problemáticos por no poseer equi-

\footnotetext{
13 “Toda la autoridad radicaba en el texto en francés. Las palabras en inglés, sujetas momentáneamente a las convenciones del francés, eran asimiladas maliciosamente. Ferron habría dicho enquébecquoisés... Era como si el autor estuviera ejecutando una venganza lúdica, desquitándose con una lengua que, como él mismo alguna vez comentara en un desenfadado e imaginativo texto sobre anglicismos, estaba "en train de faire une job au français".

14 “¿Con base en qué consideraciones éticas reencausaríamos dichas palabras en inglés a un contexto completamente angloparlante?"
} 
valentes lingüísticos o culturales, es prácticamente seguro que se transmitirá como fiel una visión equivocada de otra cultura, una versión adicional del otro que no concuerda con la realidad.

Otros traductores, como Ray Ellenwood, Betty Bednarski y Kathy Mezei, han optado más bien por una práctica inversa, es decir, por intentar dejar algo de la otredad del original, tanto a nivel lingüístico como cultural, produciendo trabajos no tan accesibles y fluidos. En ellos, la traducción se vuelve más evidente y menos "correcta" al obligar al lector a tomar una postura y a elaborar una interpretación propia. Algunas de las estrategias que han utilizado son, por ejemplo, según Mezei, cursivas, paréntesis, notas de traductor/a, adiciones, alteraciones deliberadas y frases explicativas (145). Otras veces han jugado con dos variedades del inglés o han conservado los términos ingleses pero empleando cursivas o, incluso, escribiéndolos como si los pronunciara un francés, aunque esta última opción por lo general ha sido mal recibida pues tiende a producir un efecto cómico no deseado y poco correcto políticamente hablando. Lo que queda claro en las reflexiones de muchos de estos traductores más experimentales es que no parece haber soluciones ideales. Por ejemplo, Bednarski, en su capítulo "From Ouèredéare to Soçaurez: Translating the English of Jacques Ferron", se hace las siguientes preguntas: "What to do with them in an English version? The question was unanswerable when I first asked it. And it still is. Yet its implications are far-reaching, both for an appreciation of the possibilities (and impossibilities) of translation, and for an understanding of Jacques Ferron's texts" (Simon, ed. 1995: 111). ${ }^{15}$ Acto seguido, aporta las siguientes reflexiones:

[W]ith the experience of language as it is lived by francophones in Quebec comes the awareness of a particular strangeness that is not of French itself. French writing in Quebec grows out of a linguistic environment of which English is inescapably part. Small wonder, then, that the two languages come into meaningful contact within literary texts. From the hybrid, anglicism-studded dialogues and narratives of the literature of joual to truly bilingual works like Michèle Lalonde's "Speak White," the combinations vary, but they are always politically charged. This is because language, as Sherry Simon has pointed out, is still seen as the foremost marker of cultural identity in Quebec, and because French, despite its prestige, coexists there with English in a relationship of fundamental inequality $(111-112) .{ }^{16}$

15 “QQué hacer con ellas [las palabras en inglés] en la versión inglesa? La pregunta era imposible de responder cuando me la hice por primera vez y aún lo es. No obstante, sus implicaciones son de gran relevancia tanto para apreciar las posibilidades (e imposibilidades) del acto de traducir como para comprender los textos de Jacques Ferron".

16 “"[A] partir de la manera en que experimentan el lenguaje, los habitantes francófonos de Quebec cobran conciencia de una extrañeza particular que no tiene que ver con el francés en sí mismo. En Quebec, la escritura en francés tiene lugar en un ambiente lingüístico del cual la lengua inglesa es también inevitablemente parte. Sorprende poco, por tanto, que en la escritura literaria las dos lenguas entren en contacto de forma significativa. De los diálogos y relatos híbridos, salpicados de anglicismos, que produce la literatura del joual hasta obras verdaderamente bilingües como "Speak White" de Michèle Lalonde, las combinaciones varían, pero siempre poseen una carga política. Esto se debe a que la lengua, como lo ha señalado Sherry Simon, es 
Con esto llegamos al último punto que me interesa subrayar en esta sección: el marco político e ideológico dentro del cual se dan gran parte de estas prácticas y la conciencia que de ello tienen los traductores. Para Kathy Mezei, la discusión comienza con el sólo hecho de traducir la literatura en francés de esta región al inglés, ya que esto en sí podría ser una forma de traición, una contribución a que todo vaya siendo asimilado por la cultura dominante. En su texto "Speaking White: Literary Translation as a Vehicle of Assimilation in Quebec", ${ }^{17}$ se cuestiona acerca de la labor que debieran llevar a cabo quienes traducen del francés al inglés en Canadá, considerando la susceptibilidad de los quebequenses ante la infiltración de la lengua y la cultura inglesas en su comunidad. En opinión de Mezei, dichos traductores debieran interesarse en la carga cultural que trae una lengua consigo y, por ejemplo, lograr transmitir el sentido del inglés como signo dentro del texto en francés que traducen (Simon, ed. 1995: 134). Más adelante menciona cómo la obra de autores sumamente revolucionarios y comprometidos con la identidad quebequense, como Hubert Aquin, queda descontextualizada, despolitizada y pierde toda especificidad al ser traducida profusamente al inglés o, peor aún, queda: "transformed into a figure of bland universality, and normalized within the English-Canadian scene" (135). ${ }^{18}$

Para Mezei, entonces, una de las grandes reflexiones actuales gira en torno a los costos políticos de qué, cómo y para qué se traduce literatura canadiense francófona al inglés, ya que toda esta práctica se halla situada peligrosamente entre una actividad que tanto puede diluir especificidades de la lengua y la cultura de Quebec, como, muy al contrario, fungir como vehículo para difundir justamente esta otredad y diferencia (136). Así pues, el asunto radica no sólo o no tanto en la traducción como interpretación, como traspaso de términos de una lengua a otra, sino en algo más amplio que subyace a esta práctica concreta y que se puede resumir como el análisis de lo que significa un texto traducido en relación con el contexto propio de su lengua comparado con lo que significa el texto inicial en su contexto original. Pues, como bien dice Joseph Graham, en una cita que incluye Mezei: "Translation can hardly solve the problem of meaning if any question remains about that meaning in terms that define translation itself' (137). ${ }^{19}$

Mezei concluye afirmando que lo que importa cada vez más es producir traducciones en las que el traductor pueda dejar constancia de las limitaciones y dificultades ideológicas que surgieron a la hora de realizar su trabajo, así como de las estrategias que se utilizaron, la mayoría de las cuales son el resultado de la relación que existe en

aún vista como el principal indicador de identidad cultural en Quebec y a que el francés, pese a su prestigio, coexiste con el inglés en una relación de desigualdad fundamental".

${ }^{17}$ El título mismo del artículo resulta muy significativo por la expresión "Speaking White", que aquí —al igual que el poema ya mencionado "Speak White" de Michèle Lalonde - se refiere a hablar inglés pero con toda una serie de connotaciones de poder y dominación.

18 " [... transformada en una figura de universalidad anodina y normalizada dentro de la escena anglófona canadiense".

19 "La traducción difícilmente puede resolver problemas de significado si aún prevalecen dudas con respecto al significado mismo en relación con la definición misma de traducción”. 
un lugar y un tiempo dados entre dos lenguas que conviven, muchas veces en una situación de desigualdad y, por ende, nunca del todo perfecta. ${ }^{20}$ Esta visión de la traducción como un ejercicio frecuentemente insatisfactorio, muy alejado del ideal, en el sentido de que no logra establecer una relación recíproca y balanceada entre dos lenguas, sino que muy por el contrario - y esto es parte de su desafío e importanciapone en el centro de la cuestión no sólo las diferencias entre dos sistemas culturales y lingüísticos distintos, sino también toda la compleja red de relaciones de poder entre dichos sistemas, es una de las características más importantes de los estudios de traducción hoy en Canadá.

Otro punto a mencionar es el libro destacado de Sherry Simon, Translating Montreal. Episodes in the Life of a Divided City, el cual ha conducido a un estiramiento de límites así como al enriquecimiento de las características del estudio de la traducción. ${ }^{21}$ En él, Simon explora (muchas veces haciendo comparaciones con la arquitectura) las relaciones que se establecen sobre todo entre las dos comunidades lingüísticas y culturales mayoritarias en Montreal (es decir, intranación), a través de las diversas situaciones concretas que involucran al inglés y al francés. Pues a pesar de que, oficialmente, todo se encuentra muy en orden debido a un bilingüismo equitativo, en la vida real esto no es así. La autora analiza las relaciones complejas y cambiantes que se dan entre las lenguas - y esto es lo interesante - en los productos de traducción que emergen, siendo éstos reflejo y termómetro de toda esta complicada realidad social y política. Y aquí es importante ver cómo para Simon el concepto mismo de traducción va mucho más allá de lo que normalmente entendemos como tal, ya que lo que a ella le interesa cada vez más son los procesos silenciosos e invisibles que se dan por debajo de la superficie y que consisten en trasladar conceptos, ideas, historias y acontecimientos — es decir, todo un bagaje cultural — de una comunidad a la otra. Sobre Translating Montreal, nos dice ella misma:

Because the cultural distance separating the two sides of Montreal was as vast as an ocean, the voyage across languages was a fraught venture. This book is an exploration of the changing meanings of these voyages — and the translations they produced - in the divided city. It sketches out modes of literary passage since the $1950 \mathrm{~s}$, from the then of the colonial city and the Quiet Revolution to the now of the mixed and cosmopolitan Montreal (2006: 4). ${ }^{22}$

${ }^{20}$ Lo anterior se basa en las ideas desarrolladas por André Lefevere, uno de los teóricos de la traducción más destacados de las últimas décadas en el ambiente norteamericano.

${ }^{21}$ Este texto recibió al menos dos reconocimientos: el Mavis Gallant Prize for Non-fiction y el Gabrielle Roy Prize, ambos en 2006.

22 "Dado que la distancia cultural que separa los dos lados de Montreal es tan vasta como un océano, el viaje de una lengua a otra suponía una empresa arriesgada. Este libro es una exploración del significado cambiante de dichos viajes en una ciudad dividida y de las traducciones/traslaciones que éstos produjeron. En él, asimismo, se lleva a cabo un esbozo de distintos modos de travesía literaria a partir de la década de los cincuentas, del antes correspondiente a la ciudad colonial y la Revolución Tranquila al ahora del Montreal cosmopolita y mezclado". 
Luego agrega (6-7) que su estudio se propone sacar a la luz, mediante un registro de las diversas modalidades de traducción que han surgido en la ciudad, cómo las relaciones entre las comunidades se transforman con el paso del tiempo. Y para este "viaje por las lenguas" los traductores son fundamentales, ya que son ellos quienes establecen los términos a través de los cuales las distintas realidades culturales, al replantearse las viejas fronteras, entrarán en contacto y establecerán un diálogo. Sin embargo, para Simon resulta muy claro que la experiencia de cada traductor - marcada por una historia y una coyuntura particulares - produce resultados diferentes (11), y a continuación comenta que en su estudio hay periodistas y escritores, sobre todo novelistas y poetas, quienes practican "language crossings in ways that both reflect and influence the changing relationships of the city" (idem) ${ }^{23}$ Como resultado de esas incursiones, cada "viajero" trae siempre de regreso a su propia lengua un registro de sus descubrimientos en la otra. En este punto, Simon agrega que el impulso inicial de cada viaje, de cada búsqueda, repercutirá en el tipo de material que se rescata y se traslada a la lengua primera: "the impulse that draws storytellers away from home impinges on the messages they send home" (12). ${ }^{24}$

Ahora procederé a mencionar algunos de los casos más notables de viajes entre lenguas que analiza Simon, a reserva de aclarar que aunque hay en el libro otros contenidos de gran interés, éstos no pueden comentarse aquí por razones de espacio. En primer lugar está Malcolm Reid y su libro The Shouting Signpainters de $1972 .{ }^{25}$ Para Simon, Reid se vuelve el representante más visible de un movimiento de escritores e intelectuales anglófonos de Montreal - el cual, por cierto, tuvo su comienzo en los cuarentas con otro escritor, F. R. Scott— que buscaba difundir dentro de su medio textos que permitieran conocer más y mejor a su contraparte, los montrealenses francófonos, quienes ante sus ojos poseían una serie de atributos muy atractivos. En el caso de F. R. Scott lo que tenemos es a un poeta de lengua inglesa de gran renombre que -movido por un creciente y genuino interés por lo que sucedía en el mundo de las letras en lengua francesa - se dedica a leer y seleccionar a poetas canadienses francófonos para traducirlos y difundirlos en inglés, partiendo siempre de la base de que está tratando con dos mundos, dos realidades absolutamente autónomas. ${ }^{26}$ Es por ello que

23 “[...] cruces entre lenguas que a la vez reflejan e influyen en las relaciones cambiantes dentro de la ciudad".

24 "[...] el impulso que lleva a los narradores de historias lejos de casa incide en el mensaje que envían de vuelta a casa".

${ }^{25}$ Malcolm Reid, The Shouting Signpainters: A Literary and Political Account of Québec Revolutionary Nationalism. Toronto, McClelland \& Stewart.

${ }^{26} \mathrm{Al}$ respecto, conviene citar lo que Simon dice en su artículo "The Language of Cultural Difference": "To return to the literature of the late nineteenth and early twentieth centuries is to plunge into a time when cultural difference was not so much a problem as a given - a tangible an unquestioned fact of collective reality. The 'French' and the 'English' were irremediably different, one from the other; in these differences seemed to speak the nature of human difference" $(1992,162)$. ("Regresar a la literatura de los siglos XIX y $\mathrm{XX}$ es sumergirse en un tiempo en el que la diferencia cultural no era considerada tanto un problema, sino 
sus traducciones se caracterizan por su literalidad, la cual Simon define como una “interlinear translation” (49), una traducción que busca equivalencias casi palabra por palabra y que incluye información del contexto en comentarios adicionales. ${ }^{27}$

Sin embargo, el interés de Simon se centra principalmente en The Shouting Signpainters de Reid. Ella define este texto como un "travelogue" en el que el joven autor, lleno de admiración y entusiasmo, recorre la parte este de Montreal y registra la vida y el pulso de su comunidad francófona durante el verano de 1966, un verano caracterizado por la marcada politización de este sector de la población en pleno proceso de revisar su relación con la sociedad anglófona y recuperar así una dignidad lastimada. De hecho, para Simon, el testimonio que ofrece el libro de este nuevo territorio ideológico lo convierte en "a model of translation as discovery that remains unsurpassed in intensity" (12). ${ }^{28} \mathrm{Y}$ esto es justamente lo que la lleva a utilizarlo como el punto de partida de su estudio. El viaje en este caso consta de un recorrido por, y un registro de, el mundo de los escritores parti pris, ${ }^{29}$ un mundo en donde lo cultural y lo político se potenciaban mutuamente. Algunos de estos escritores son Jacques Ferron, Hubert Aquin, Jacques Renaud y Gaston Miron, y lo que hace Reid es presentar a cada uno de ellos y dar un panorama lo más completo posible tanto del contexto en el que escriben como de todas las discusiones, encuentros y desencuentros que surgen dentro de dicho grupo (29-31). Y para escribir este viaje Reid debe tomar en cuenta tanto el uso del joual como de una serie de términos pertenecientes al universo del marxismo, el psicoanálisis y el existencialismo francés, los cuales resultan fundamentales dentro del discurso de estos escritores, y buscar cómo transportarlos al inglés dentro de su libro. De no haber proporcionado estas referencias, el autor no habría logrado transferir la realidad ajena que tanto lo apasionaba a su propio medio y, para lograr este cometido, Reid incluso elabora un glosario.

Simon acaba este primer capítulo (56) haciendo varias reflexiones de interés, en las que, ante todo, reconoce el papel tan importante que desempeñó Reid al descubrir para el Canadá anglófono este mundo vivo, novedoso y muy diferente, a través de una crónica en la que, de modo cuidadoso, el autor busca hacer entendible lo distinto. También comenta cómo Reid pasó de traductor a converso y ya no regresó al Montreal inglés. No obstante, su obra abrió un camino que luego habrían de transitar infinidad de otros traductores, quienes en años posteriores y hasta la fecha han buscado trans-

un dato, un hecho tangible e incuestionable de la realidad colectiva. Lo 'francés' y lo 'inglés' eran irremediablemente diferentes uno del otro y la naturaleza de la diferencia humana parecía hablar a través de estas diferencias".)

${ }^{27}$ Simon menciona dos libros publicados por F. R. Scott como traductor: St-Denys Garneau \& Anne Hébert: Translations / Traductions. Vancouver, Klanak Press, 1962, y Poems of French Canada. Burnaby, Blackfish Press, 1977.

28 "[...] un modelo de traducción como descubrimiento que no ha sido superado en intensidad".

${ }^{29}$ Llamados así por la revista homónima donde publicaban, la cual tenía una posición considerada muy radical en torno a la situación de opresión que, según estos autores, padecía su comunidad. La revista se publicó de octubre de 1963 a octubre de 1968. 
portar, de diversas maneras y resultados variados, la escritura francesa de Montreal en particular, y de Quebec en general, al contexto anglófono.

El siguiente estudio gira en torno a otro "viajero": A. M. Klein, un poeta de Montreal de origen judío, es decir, un escritor que se ubica fuera de las dos comunidades culturales principales de dicha ciudad, pues pertenece ya a uno de los varios grupos de inmigrantes (como los polacos, griegos e italianos) que fueron llegando y que complejizaron la dinámica local al agregar y poner en circulación nuevos ingredientes simbólicos y lingüísticos. Su importancia, entonces, radica justamente en esta pertenencia a un grupo incluso más otro que el francófono para el "establishment" angloparlante y en sus intentos por incorporar las características principales de su legado cultural al Montreal que le tocó vivir. Simon es muy clara cuando describe la importancia de la tarea realizada por Klein: "This was not a simple task of mediation (of informing one linguistic group of the achievements of another); nor was it an attempt to translate North American Jewish experience out of or away from the past. His goal was to imbue the present with the forms and styles of the past, to express a culture traversed by many languages and histories" $(61) .^{30}$

Y esto para Klein implicó, como acertadamente dice Simon, no traducir elementos yiddish o incluso hebreos al inglés, sino un proceso mucho más complejo. Su objetivo era trasladar al inglés del Montreal de su tiempo rasgos lingüísticos y culturales pertenecientes al mundo de la inmigración judía de sus padres, pero de modo que se incorporaran dentro del tejido anglófono para dejar constancia de la hibridez de su momento histórico. Es más, buscaba que elementos significativos para él tanto del yiddish como del hebreo, e incluso a veces del francés, interactuaran de manera simultánea dentro de un texto, dejando ver no sólo la mezcla cultural y lingüística que caracterizaba su entorno, sino la relevancia que seguían teniendo todas estas presencias, supuestamente pasadas, en el presente de esa comunidad montrealense de inmigrantes, procedimiento que lo alejaba completamente de cualquier proceso de traducción convencional (62-63).

Sin embargo, estas incursiones al pasado-presente judío y sus experimentos a la hora de intentar dejar registro de la marca de lo uno en lo otro - por cierto, muy alejadas de lo que podría simplemente ser la manera contaminada de expresarse en inglés de un inmigrante judío - son de un enorme interés y han sido estudiados crecientemente en relación con los grupos de literatura de vanguardia (modernism en inglés) de la época. A continuación cito a Simon en extenso por considerar muy iluminador lo que dice sobre este impulso tan particular que marca la obra de Klein:

\footnotetext{
30 "Esto no constituía una simple tarea de mediación (de informarle a un grupo lingüístico acerca de los logros de otro); tampoco se trataba de un intento por traducir la experiencia judía norteamericana fuera de o lejos del pasado. Su objetivo era imbuir el presente con las formas y estilos de antaño, expresar una cultura atravesada por numerosos lenguajes e historias".
} 


\section{$198 \square$ LA TAREA (CADA VEZ MÁS IMPROPIA) DEL TRADUCTOR}

This dialectic in some ways makes Klein a failed translator. If successful translation is replacing one coherent linguistic system by another, Klein never attained this goal. If the desire of the immigrant writer is to assimilate into the mainstream tradition, then he was not a typical immigrant writer. His strategy was to remain between languages, forcing the limits of English, subjecting it to the strain of alien vocabulary and rhythm. This means that Klein was sometimes considered obscure and intentionally difficult (63). ${ }^{31}$

En suma, Simon en su estudio destaca que al ser habitante de Mile End, un barrio de inmigrantes en Montreal, Klein hizo confluir varias lenguas en el inglés, incorporando a dicho idioma la memoria cultural hebrea y yiddish e, incluso, elementos del francés, lo cual pone de manifiesto la cercanía emocional que mantenía con su ciudad. Su propósito era conformar un lenguaje para la modernidad judía marcada por la experiencia de la diáspora. Por su carácter híbrido e imaginativo, sus escritos conservan aún ahora su audacia como experimentos literarios que capturan la variedad lingüística y cultural de la vida en Montreal (13). ${ }^{32}$

En el capítulo tercero de Translating Montreal, Simon traza la ruta por medio de la cual el yiddish, que en algún momento fuera la tercera lengua más importante de la ciudad, continuó introduciéndose en la vida literaria montrealense. La autora señala que si bien la lengua de contacto con el yiddish fue tradicionalmente el inglés, en algún momento surgieron traducciones directas del yiddish al francés, lo cual supuso un cambio importante en la territorialización intelectual de la ciudad (14). En relación con lo anterior, menciona el caso de Pierre Anctil, antropólogo, historiador y traductor, quien se ha esforzado — no de manera aislada, sino como miembro de un movimiento cultural más amplio que tuvo su comienzo en los ochentas - por estrechar los lazos entre la esfera francófona del Montreal actual y el legado histórico de la comunidad judía. La labor de Anctil destaca ya que, sin ser él mismo de origen judío, aprendió yiddish y sus traducciones abarcan los géneros de la poesía, la novela, la autobiografía y la historia. Este tipo de contribuciones han permitido que surja una nueva vía de comunicación y contagio entre culturas que, a su vez, ayuda a contrarrestar la hegemonía cultural de la lengua inglesa (96).

Sin embargo, los "viajeros" cuyas travesías culturales y literarias más llaman la atención de Simon son autores como Gail Scott, Erin Mouré, Jacques Brault, Michel Garneau, Agnes Whitfield y, de nuevo, Nicole Brossard, quienes han estirado los lími-

31 "Esta dialéctica hace que Klein sea, de diferentes maneras, un traductor fallido. Si una traducción exitosa es aquélla en la que un sistema lingüístico coherente es reemplazado por otro, Klein nunca alcanzó dicha meta. Si el deseo del escritor inmigrante es asimilarse a la tradición dominante, entonces él no fue un escritor inmigrante típico. Su estrategia consistió en permanecer entre distintas lenguas, forzando los límites del inglés y sometiendo éste a la sonoridad de un vocabulario y un ritmo ajenos. Debido a lo anterior, Klein era a veces considerado un autor oscuro e intencionalmente difícil".

${ }^{32}$ Recientemente se ha comenzado a traducir a Klein al francés, lo cual ha ocasionado un creciente interés por parte de la crítica francófona respecto de la trascendencia de dicho autor (14). Ver A. M. Klein, Le second rouleau. Trad. de Charlotte y Robert Melançon. Quebec, Boréal, 1990. 
tes tradicionales de la traducción y llevan a cabo una suerte de "interferencia creativa" tanto en los territorios del inglés como en los del francés. En lugar de intentar trasladar de manera eficiente un texto de una lengua a otra, estos autores utilizan el contacto, la relación entre lenguas para otros fines más nuevos, inesperados, desobedientes (119). Así pues, Simon ofrece un panorama de algunos de los procesos de traducción que se llevan a cabo actualmente en Montreal:

[...] processes that reflect the crossovers of an increasingly mixed and global city. They are extensions of the innovative language relations that characterize activities of the everyday, as well as the interlingual practices that emerge in theatre, cinema and advertising. Like postmodern architecture, these practices separate structure from function, turning fragments of history to new uses. Translingual poetics, creative interference, "non-translation," "transelation," 33 and "translation without an original" are some of the techniques that drive writing in both English and French. These are the "warm" forms of translation, ${ }^{34}$ which turn translation away from its normative function, disturbing the boundaries of each cultural space. They speak of the contact zones of the city today, where fluid interactions between languages and adulterated forms of speech make conventional transfer impossible. Gail Scott, Erin Mouré, Jacques Brault, Michel Garneau, Nicole Brossard, and Agnes Whitfield use a second language as an impulse and element of their own language (14).$^{35}$

No obstante, aunque las formas de traducción mencionadas en la cita previa destacan por su carácter provocativo, es justo decir que en Montreal ya existe hoy un cierto grado de "intercomprensión" entre la comunidad de habla francesa y la de habla inglesa, lo cual quiere decir que hablar de traducción convencional sale, hasta cierto punto, sobrando. Por esta razón, en muchas situaciones (discursos oficiales, textos de presentación) lo que se utiliza cada vez más es que se pase de una lengua a otra

${ }^{33}$ Término acuñado por Erin Mouré al traducir a Fernando Pessoa (Alberto Caeiro) y que, al parecer, combina la palabra translation (traducción) y elation (euforia). La respuesta de Mouré a la poesía de Pessoa es altamente sensitiva y ella misma describe el proceso de traducción como uno de "exceso" (152-53). El caso de Mouré se comentará más adelante.

${ }^{34}$ Simon habla de las formas "frías" de traducción, las cuales reafirman una distancia permanente entre dos comunidades lingüísticas y un respeto por lo diferente, mientras que las formas "cálidas" contemplan un mayor grado de compromiso personal y de transformación (40-41).

35 “[...] procesos que reflejan los entrecruzamientos que tienen lugar en una ciudad cada vez más mezclada y global. Dichos procesos son extensiones de las innovadoras relaciones lingüísticas que caracterizan las actividades cotidianas, así como las prácticas que emergen en el teatro, el cine y la publicidad y en las que se emplea más de una lengua. Tal como sucede en la arquitectura posmoderna, estas prácticas disocian estructura y función, toman fragmentos de la historia y les dan nuevos usos. La poética de lo translingüe, la interferencia creativa, la "no-traducción", la "transelation", y la "traducción sin original" son algunas de las técnicas que rigen el escribir en inglés y francés a la vez. Éstas son las formas "cálidas" de traducción, las cuales alejan el acto de traducir de su función normativa, sacudiendo las fronteras de cada espacio cultural y poniendo de manifiesto las zonas de contacto que existen en la ciudad actualmente, donde las interacciones que se dan de manera fluida entre distintas lenguas y las formas adulteradas del habla hacen que una transferencia de tipo convencional sea imposible. Gail Scott, Erin Mouré, Jacques Brault, Michel Garneau, Nicole Brossard y Agnes Whitfield emplean una segunda lengua como impulso y un elemento de su propia lengua”. 
sin necesariamente repetir de modo literal lo que se ha dicho, con lo cual el hablante bilingüe aprovecha el 100\% del mensaje (121).

Simon comienza el capítulo cuarto del libro comentando el caso de la escritora Gail Scott, ${ }^{36}$ quien en su artículo "My Montréal. Notes of an Anglo-Québécois Writer"37 narra un paseo por esta ciudad con un acompañante estadounidense, al que instruye acerca de la historia y las costumbres tan particulares de este sitio bicultural. Sin embargo, lo principal para Simon es cómo en el texto se transluce el sentimiento de que esta condición bilingüe más que un impedimento se ha vuelto un incentivo, de modo que Scott ve la ciudad como un laboratorio donde ejercitar la imaginación en más de una lengua a la vez (124). Luego Simon agrega:

Her aim, as an English writer, is not to find a comfortable vernacular, but to do something quite the opposite: to write against ordinary language in order to gain critical purchase and a stronger consciousness of language as an organizing system. The presence of French helps. It will allow her to construct an idiom that is slightly inflected, that slows down the rhythm of recognition (125). ${ }^{38}$

Entonces, a diferencia de lo que ocurrió con los escritores de generaciones anteriores como F. R. Scott, ${ }^{39}$ quien escribía firmemente posicionado dentro del contexto de la lengua inglesa, esta escritora más joven representa una nueva etapa en la relación entre el inglés y el francés, la cual le permite transportarse de manera novedosa a otra dimensión cultural y lingüística. Su objetivo final es revisar, desde otra perspectiva, su propia y compleja realidad anglófona en el escenario de una ciudad dividida y crear, a través de un nuevo lenguaje - lo mismo que habían hecho a su modo las feministas para sí mismas - , una nueva identidad anglo-quebequense (126). Y esta nueva identidad se caracteriza por una dualidad muy específica. Sobre esto Simon comenta:

${ }^{36}$ Es autora de la novela Heroine (Toronto, Coach House Press, 1987), la cual fue traducida al francés por De Lotbinière-Hardwood y publicada como Héroïne (Montreal, Editions du Remue-ménage, 1988). En 1993 publicó la novela Main Brides: Against Ochre Pediment and Aztec Sky (Toronto, Coach House Press), traducida al francés como Les fiancées de la Main por Paule Noyart (Montreal, Leméac, 1999), y en 1999 publicó My Paris (Toronto, Mercury Press), novela escrita a manera de memorias. Véase también su cuento "Burro andando", publicado en la antología Otras voces canadienses (México, UNAM, Difusión Cultural, 2009).

${ }^{37}$ Publicado originalmente en 1998 en la revista Brick, núm. 59, pp. 4-9.

38 "Su objetivo, como una escritora en lengua inglesa, no es encontrar una lengua vernácula cómoda, sino hacer casi lo opuesto: escribir en contra del lenguaje ordinario con el propósito de obtener un asidero crítico y una conciencia más firme del lenguaje como un sistema organizativo. La presencia del francés ayuda. Le permitirá construir un lenguaje un tanto flexivo, que desacelera el ritmo en el que se lleva a cabo el reconocimiento".

${ }^{39}$ Otros ejemplos mencionados por Simon son Morley Callaghan, Louis Dudek, el propio A. M. Klein, Irving Layton, Mavis Gallant, Brian Moore, Mordecai Richler y Leonard Cohen, quienes aunque tienen una íntima conexión con Montreal, retratan una ciudad en la que la comunidad francófona desempeña mayormente el papel de espectadora (125). 
[...] "writing over the cusp between Quebec's two main language groups" takes "a certain rigour of intention"... In order to account for the Quebec of today, Scott explains that she felt obliged to "participate in and ultimately address, two often clashing, but also mutually nourishing cultures, simultaneously. [...] French language and culture in a sense also belong to me; it is part of my cultural background, makeup" (idem). ${ }^{40}$

La escritura de Scott busca situarse en la encrucijada entre dos culturas que, ya en esta época, no pueden realmente separarse de manera clara. Lo anterior implica utilizar una doble lengua, un inglés en donde el francés - necesario para expresar aspectos de su compleja realidad bicultural — se hace presente con bastante frecuencia (de modos más o menos obvios), desequilibrando el texto de modo muy estimulante. El resultado son ejercicios de "cruzamiento de lenguas" (language crossing) que escapan del ámbito de la traducción convencional, pues en sí son parte intrínseca y temporalmente diacrónica del texto original y no pertenecen a ninguna versión previa. De acuerdo con Scott, todo resabio de traducción que pueda tener su escritura se debe al hecho de que ésta registra un paso anterior de translación de la "otredad" francófona quebequense a la sensibilidad y conciencia de la escritora anglófona, creando una nueva realidad más intermedia e indefinida. ${ }^{41}$

El caso de Agnes Whitfield ${ }^{42}$ también resulta de interés. Esta escritora, si bien es de extracción anglófona, motivada por sus experiencias como traductora a la lengua francesa, acabó por abandonar el inglés y escribir de manera creativa en francés. Sobre este proceso de transfiguración o desplazamiento, Simon dice lo siguiente: "The translator has herself been transformed. She has entered the dance of language and found herself carried away by its rhythms. Though she was meant to be the purveyor, she ends up herself purveyed into the language of the original. She travels a reverse route, into a

40 “[...] 'escribir sobre el vértice donde confluyen los dos principales grupos lingüísticos de Quebec' requiere 'un cierto rigor de intención'. [...] Con el propósito de dar cuenta del Quebec de hoy día, Scott explica que se sintió obligada a 'participar en y, en última instancia, interpelar simultáneamente a dos culturas que con frecuencia chocan, pero que también se alimentan mutuamente. [...] La cultura y la lengua francesas, de alguna manera, también me pertenecen; son parte de mi bagaje cultural, de mi carácter"”.

${ }^{41}$ Vale mucho la pena comentar, aunque brevemente, el concepto-imagen de la "coma de la traducción" propuesto por Scott. En el paseo por la ciudad que narra en "My Montreal", la autora llega al puente Jacques Cartier, cuyo arco semeja una "coma" que "puntúa" el horizonte. Tanto el puente como la coma, nos dice Simon, articulan secciones que, de otro modo, estarían separadas (126). Ambos son, asimismo, puntos de conexión que permiten el doble tránsito de una lengua a otra y su importancia para Scott radica en que, en lugar de pretender ocultar el proceso mismo de traducción o transición, ponen de manifiesto el momento mismo del cruce y, con ello, se explicitan las diferencias entre una lengua y otra. El puente se vuelve, así pues, en un espacio de la conciencia y en el espacio virtual donde acontece la escritura (131).

${ }^{42}$ Es autora del libro de poesía $O$ cher Emile, j'e t'aime. Traductions sans original (Hearst, Le Nordir, 1993) y del relato Où dansent les nénuphars (Ottawa, Le Nordir, 1995). También es editora del libro de 2005 Le métier du doble. Portraits de traductrices et traducteurs littéraires (Montreal, Fides) y de Writing Between the Lines: Portraits of Canadian Anglophone Translators (Waterloo, Wilfrid Laurier University Press) publicado en 2006. 


\section{$202 \square$ LA TAREA(CADA VEZ MÁS IMPROPIA) DEL TRADUCTOR}

language she transforms from second to first" (142). ${ }^{43}$ Simon agrega que en este caso, y debido al contexto tan particular que encontramos en Montreal, lo que sucede es que la segunda lengua responde a experiencias intelectuales, lingüísticas y afectivas vividas por Whitfield, anteriormente, en una primera lengua. Sin embargo, cuando la autora afirma que "sólo puede escribir a través de la traducción", queda claro que aunque el francés le permite moverse fuera de los límites de su identidad originaria, siempre habrá algo de ésta presente en su escritura. Es decir, nunca pertenecerá plenamente a un lado u otro de la frontera lingüística y cultural (143).

Otra viajera es Erin Mouré, quien, incluso para Simon, estira los límites de la traducción al máximo y ha iniciado todo un nuevo tipo de prácticas y experimentos que han comenzado a hacerse cada vez más frecuentes en Montreal. Su ejercicio más comentado fue la versión que elaboró de Poesias de Alberto Caeiro y que Mouré tituló Sheep's Vigil by a Fervent Person $(2001)^{44}$ y que no califica como "translation" sino como "transelation". Mouré — de antecedentes gallegos- se ha interesado crecientemente por las múltiples lecturas que todo poema contiene, así como por la obra de Pessoa, con su gran variedad de heterónimos. Lo que motivó su traducción, decididamente sui generis, de este escritor es considerar que las múltiples identidades que encierra la obra del portugués le permiten a ella sumarse al proceso de creación poética, vertiendo partes de esta obra al inglés. No obstante, sus versiones son sumamente libres y creativas, lo cual es resultado de las transformaciones que sufre ella misma $^{45}$ al entrar en contacto con estos escritos, un contacto que ella describe como absoluto y sin límites. Acerca de la práctica de "transelation", Mouré ha dicho lo siguiente:

I started "translating" without goal and without aim. Making words in English to incarnate, alongside the text, my own surprise and pleasure, and my own readerly sitedness in time and culture. To make this siting not just visible, but a propulsive, gestural element in the new text. Bringing Pessoa's Caeiro from his Portuguese hilltop into Toronto, Canada, into the patient "rural" that still thrives just under its urban surface (apud Simon: 153) ${ }^{46}$

43 "La traductora ha sido ella misma transformada. Ingresó a la danza de la lengua y se dejó llevar por sus ritmos. Aunque su intención era ser la transmisora, terminó ella misma siendo transmitida a la lengua del original. Whitfield viaja en reversa no a una segunda, sino a una primera lengua".

${ }^{44}$ Quizá no está de más recordar que la palabra portuguesa pessoa significa person en inglés y persona en español.

${ }^{45}$ La traductora, en este caso, opta por cambiar su nombre de Erin a Eirin, la forma galaica de su nombre, secundando así el uso de heterónimos del propio Pessoa (152).

46 “'Comencé a 'traducir' sin objetivo ni intención. Las palabras en inglés encarnaban, junto con el texto, mi propia sorpresa y placer, así como mi propia ubicación como lectora en un tiempo y una cultura. Buscaba que esta ubicación no sólo fuera visible, sino que se convirtiera en un elemento propulsor y gestual en el nuevo texto; buscaba transportar al Caeiro de Pessoa de su cima en un cerro en Portugal a Toronto, Canadá, a la paciente 'ruralidad' que aún crece justo por debajo de su superficie urbana". 
Y en otro texto agrega que, al traducir los poemas de Alberto Caeiro, buscaba establecer un proceso de traducción que capturara realmente la "multiplicación de gestos" que conforman la "obra en Pessoa". "Each Pessoa line provokes an Eirin line and sometimes the Eirin line provokes a further line, an exhorbitance of gesture, a multiplication of Pessoan effect and affect. As a translator, I just let the process of excess operate, freely but with attention" (apud Simon: idem). ${ }^{47}$

Simon ofrece una serie de comentarios adicionales acerca de este ejercicio de "transelation": comenta que al leer a Mouré lo que sucede es que nos vamos habituando a ir y venir entre lo que se ve y lo que no, lo que existe en una lengua y lo que se puede producir en la otra, para así echar a andar una práctica poética en donde lo que existe funge como impulso para lo que puede ser creado (154). El resultado es un libro bilingüe en donde se presenta la versión original en portugués junto a la versión de Mouré - lo cual apunta aún a una división, separación entre una cosa y la otra—, una versión que reubica a Pessoa en el Toronto de hoy, si bien, como insiste Mouré, el Pessoa de fondo sigue siendo el mismo. Aunque en Sheep's Vigil by a Fervent Person Pessoa es llevado a Toronto, donde vive una vida pseudopastoral en lo que le queda de pastoral a Toronto bajo la superficie, la traducción de Mouré, de acuerdo con ella misma, conserva la estructura del texto original: "The prior text accelerated, encouraged the translatory work, even to the point of altering its translator [...]. I see this book as translation, as faithful even if different. That's why it appears in a bilingual edition with the Portuguese originals - my deflections of Pessoa's texts are thus visible, even if you do not read Portuguese" (apud Simon: idem). ${ }^{48}$

Finalmente, es importante mencionar aquí a la poeta Anne Carson, ${ }^{49}$ quien ha llegado a hablar de las "traducciones sin un original" ${ }^{50}$ con lo cual hace referencia a todos aquellos casos en los que, debido a los huecos o lagunas en una lengua, se utilizan expresiones torpes, no del todo felices, para nombrar algunas de las complejas realidades que se gestan en, por ejemplo, una ciudad tan múltiple como Montreal. En este tipo de contextos, una lengua carga con la marca, la presencia de otra lengua, y las compensaciones lingüísticas que se llevan a cabo por dicho motivo dan la impresión de que se está realizando una traducción no del todo apropiada, lo cual nos conduce

47 "Cada verso de Pessoa provoca un verso de Eirin y, en ocasiones, el verso de Eirin provoca un verso adicional, una exhorbitancia gestual, una multiplicación del efecto y afecto de Pessoa. Como traductora, sólo dejo que este mecanismo de excesos opere libremente, no sin dejar de prestarle atención".

48 "El texto original aceleró, alentó el trabajo de traducción hasta llegar al punto de alterar a la propia traductora $[\ldots]$. Considero que ésta es una traducción fiel a pesar de ser diferente. Es por ello que aparece en una edición bilingüe que incluye los originales en lengua portuguesa, de modo que mis desviaciones de la obra de Pessoa son visibles, incluso si el lector no puede leer en portugués".

${ }^{49}$ Nacida en 1950, es poeta, traductora y catedrática. Vivió algunos años en Montreal. Por el volumen de poesía The Beauty of the Husband (Toronto, Vintage Canada) de 2001 le fue otorgado el T. S. Eliot Prize y por Men in the Off Hours de 2000 (Nueva York, Alfred A. Knopf), el Griffin Poetry Prize.

${ }^{50}$ Expresión tomada de su experiencia como estudiosa de textos clásicos, en donde desarrolló el hábito de que, en caso de duda o dificultad al leer una traducción de un texto griego, veía la página izquierda para cotejar con el original. 
gradualmente a reflexionar acerca de cómo las distintas lenguas no son nunca equivalentes. Pero, aún más importante, lo anterior nos invita a meditar en torno a los universos que quedan fuera de las fronteras que establecen las distintas lenguas, incluso de la lengua en su sentido más amplio y de las búsquedas que realizan algunos viajeros incansables en su intento por explorar dichos universos (218).

Para concluir esta sección quisiera citar nuevamente a Sherry Simon en un comentario sobre cómo las lenguas de la ciudad nutren la imaginación literaria de sus escritores:

In the divided city, "perverse" forms of translation are at home. They confirm the imaginative role played by the city itself. This is not the same as to repeat what has often been said of English Montreal writers, for instance, that they experience a situation of "double exile" (Solway 2003) and therefore both suffer — and benefitfrom a double sense of marginality ... or of French-language writers, that they find themselves at an uncomfortable crossroads between the English-language populations of America and the French of the continent. "Perverse" translations propose a different diagnosis, suggesting that the work of Montreal writers is the result not of isolation but of a special kind of contact. It is contact and interaction (not isolation and exclusion) that fuel the work of many Montreal writers - in English and in French $(160) .{ }^{51}$

A modo de conclusión general, quisiera subrayar una vez más cómo los estudios de Sherry Simon sobre las distintas formas que han ido caracterizando a la traducción en Canadá en años recientes vuelven visibles una serie de ejercicios y postulados novedosos que obligan a repensar numerosos planteamientos tradicionales ligados tanto con la práctica como con la teoría de la traducción.

\section{Obras citadas}

Simon, Sherry. 2006. Translating Montreal. Episodes in the Life of a Divided City. Montreal: McGill-Queen's University Press. 1996. Gender in Translation. Cultural Identity and the Politics of Transmission. Londres / Nueva York: Routledge.

51 "Una ciudad dividida es un medio propicio para el surgimiento de formas 'perversas' de traducción, las cuales confirman la función imaginativa que la ciudad misma desempeña. Esto no equivale a repetir lo que con frecuencia se ha dicho acerca de los escritores anglófonos de Montreal, por ejemplo, que viven en una situación de 'exilio doble' (Solway: 2003) y que, por tanto, sufren y se benefician, a la vez, de una doble condición de marginalidad... o lo que se ha dicho de los escritores en lengua francesa, que se hallan a sí mismos en una incómoda encrucijada entre las comunidades angloparlantes de América y las francófonas de Europa. Las traducciones 'perversas' ofrecen un diagnóstico distinto y sugieren que la obra producida por los autores de Montreal es, no el resultado del aislamiento, sino de una suerte de contacto. Son el contacto y la interacción (no el aislamiento y la exclusión) los que avivan el trabajo de muchos escritores montrealenses, tanto en inglés como en francés". 
ed. 1995. Culture in Transit: Translating the Literature of Quebec. Montreal: Véhicule Press.

1992. "The Language of Cultural Difference. Figures of Alterity in Canadian Translation". Rethinking Translation: Discourse, Subjectivity, Ideology. Ed. L. Venuti. Londres / Nueva York: Routledge. Pp. 159-176. 